\title{
Adiponectin Receptor 1 Single Nucleotide Polymorphism Is Highly Associated with Hypertriglyceridemia in Asian Male-A Novel Genetic Screening to Reduce Risk of Cerebrovascular Disease
}

\author{
William C. W. Huang1, Rong-Fu Chen ${ }^{2}$, Yu-Ying Yang ${ }^{3 *}$ \\ ${ }^{1}$ Department of Family Medicine, Show-Chwan Memorial Hospital, Taiwan \\ ${ }^{2}$ Department of Medical Research and Development, Show-Chwan Memorial Hospital, Taiwan \\ ${ }^{3}$ Department of Clinical Laboratory, Show-Chwan Memorial Hospital, Taiwan \\ Email: ${ }^{*}$ claire0506@gmail.com
}

Received 20 July 2015; accepted 14 September 2015; published 18 September 2015

Copyright (C) 2015 by authors and Scientific Research Publishing Inc.

This work is licensed under the Creative Commons Attribution International License (CC BY). http://creativecommons.org/licenses/by/4.0/

cC) (i) Open Access

\section{Abstract}

Background: Adiponectin is involved in regulating both glucose and fatty acid. Associations of the known adiponectin receptors 1 (ADIPOR1) single nucleotide polymorphism (SNP) with diabetes have been demonstrated while hypertriglyceridemia is frequently associated with cerebrovascular disease (CVD) among diabetes. Triglyceride metabolism was also reported to be different between genders and estrogen was observed to interfere with adiponectin effects via ADIPOR1. It seems important to investigate whether the ADIPOR1 SNP variants may be significant determinants in triglyceride metabolism and hence be a risk of CVD in specific gender. Methods: A survey was performed on random self-reported healthy subjects aged 35 and above with their biochemical data collected. Genotyping for ADIPOR1 SNP (rs1342387) was carried out using TaqMan Genotyping Assays. Interviews were also conducted regarding stress, adverse diet behavior and exercise. Multivariable logistic regression analyses were performed to identify the strongest contributing variables. Findings: The ADIPOR1 minor allele carrier (T/T and T/C) had significantly $(p=$ 0.02) higher TG mean compared to homozygous $(\mathrm{C} / \mathrm{C})$ major alleles. TG difference was significantly higher in male $(p=0.02)$ with a larger difference in mean, whereas the difference disappeared among female $(p=0.32)$. Multivariate logistic regression analyses were performed by defining abnormal TG based on NCEP criteria of metabolic syndrome, and when all life style variables were entered in the model with ADIPOR1, only the ADIPOR1 in male showed significant ( $p$

${ }^{*}$ Corresponding author.

How to cite this paper: Huang, W.C.W., Chen, R.-F. and Yang, Y.-Y. (2015) Adiponectin Receptor 1 Single Nucleotide Polymorphism Is Highly Associated with Hypertriglyceridemia in Asian Male-A Novel Genetic Screening to Reduce Risk of Cerebrovascular Disease. World Journal of Neuroscience, 5, 323-327. http://dx.doi.org/10.4236/wjns.2015.55029 
$=0.03)$ and very high association with abnormal $\mathrm{TG}(\operatorname{Exp}(\beta) 16.31)$. Discussion: The findings provide sample evidence of a relation between ADIPOR1 SNP minor allele carrier and high TG concentrations in male. The association of abnormal TG and ADIPOR1 is much stronger than that compared to life style. The implications of this survey may be further extend to identifying the genetic risk of abnormal TG at young age and reduce the CVD incidence by early intervention.

\title{
Keywords
}

\author{
ADIPOR1 SNP, Hypertriglyceridemia, Male
}

\section{Introduction}

Hypertriglyceridemia is frequently associated with type 2 diabetes mellitus [1], and demonstrates significantly associated with lacunar infarction among diabetic patients [2]. Adiponectin is involved in regulating both glucose and fatty acid. In the Diabetes Prevention Program (DPP), lower adiponectin concentration was associated with increased diabetes risk [3] while adiponectin receptor 1 (ADIPOR1) single nucleotide polymorphisms (SNPs) were further identified to be the key of the association [4]. It seems important to determine whether the ADIPOR1 SNP may be significant determinant in triglyceride (TG) level, hence be associated with the diabetes and cerebrovascular disease.

Given the studies regarding differences in TG metabolism between male and fenale [5] as well as those indicating that estrogen interferes with ADIPOR1 [6], it is also essential to research the gender specific role of ADIPOR1 in TG metabolism. Lancet recently also reminded investigators of the importance of "analyzing data by sex, not only when scientifically appropriate, but also as a matter of routine” [7].

Because we were unaware of any previous study of this nature, the present study was designed to investigate the capacity of using ADIPOR1 SNP to identify unacknowledged metabolic abnormality among healthy subjects and across gender.

\section{Material and Methods}

The epidemiologic study of this preliminary investigation was conducted in Taiwan and performed on random subjects aged 35 and above who claimed to be healthy and under no medication. This study was approved by the Show-Chwan Memorial Hospital IRB board (IRB: \#1020602) and had been carried out in accordance with the principles of the Declaration of Helsinki as revised in 2008. Blood pressure was measured sitting, after a minimum rest of 5 minutes and values were averaged by three different measurements separated by 2 minutes from each other. Concentrations of plasma glucose, TG, cholesterol, high density lipoprotein and low density lipoprotein were determined after an overnight (12-hour) fast.

ADIPOR1 SNP variant (rs1342387) locates on chromosome 1 and code for mRNA (NM_001290553.1) intron region (http://www.ncbi.nlm.nih.gov/snp), was reported by Mather et al. to have the most statistically significant association of increased diabetes incidence $(p$-value $=0.005)$ for minor allele carriers [4]. Genomic DNA was isolated from heparin-anticoagulated blood samples using MagNA Pure LC Isolation Kit ${ }^{\circledR}$ running on the MagNA Pure LC'M (input/output volume: 200/100 $\mu$ l). Samples were first stored under $4^{\circ} \mathrm{C}$ and transferred to laboratory center within three hours. Genotyping forADIPOR1 were carried out in a laboratory center using TaqMan SNP Genotyping Assays (Applied Biosystems, Foster City, CA, USA). The probes were labeled with the TaqMan fluorescent dyes VIC and FAM, respectively. The PCR was carried out in a total volume of $15 \mu \mathrm{L}$ using the following amplification protocol: denaturation at $95^{\circ} \mathrm{C}$ for 10 minutes, followed by 40 cycles of denaturation at $94^{\circ} \mathrm{C}$ for 20 seconds, followed by annealing and extension at $60^{\circ} \mathrm{C}$ for one minute. After the PCR, the genotype of each sample was determined by measuring the allele-specific fluorescence in the ABI Prism 7500 Sequence Detection System, using SDS 1.1 software for allele discrimination (both Applied Biosystems). The PCR products were also validated by assessing the restriction fragment length polymorphism in agarose gel electrophoresis.

A total of 116 individuals, 67 females and 49 males were enrolled. The ADIPOR1 SNP allele frequency assessment satisfied the Hardy-Weinberg equilibrium [8] and may be carried through generations (chi-square test, 
$p$-value $>0.05)$ with a homozygous minor allele $(\mathrm{T} / \mathrm{T})$ of $9.4 \%$, heterozygous $(\mathrm{C} / \mathrm{T})$ of $52 \%$ and homozygous major allele (C/C) of 38.5\%. Population was categorized in two groups based on ADIPOR1 SNP variants, minor allele carrier (T/T, T/C) and the homozygous major allele (C/C). Independent sample t-tests were performed for continuous variables, and sex proportions were expressed as percentages and compared with the chi-square test in both genetic groups. We further tested the data for males and females separately to compare gender difference.

A structured interview was also conducted by trained nurses regarding stress, adverse diet behavior (http://health99.hpa.gov.tw/OnlinkHealth/OnlinkHealth_list.aspx) and exercise

(http://www.hpa.gov.tw/BHPNet/Web/Easy/FormCenterShow.aspx?No=201201130001) according to the questionnaire provided by Health Promotion Administration in Taiwan. In order to ascertain the genetic variants and life styles that contribute to lipid metabolism, we performed multivariable logistic regression analysis (adjusting for ADIPOR1, stress, adverse diet behavior and exercise) to identify the strongest contributing variables. TG was categorized into abnormal ( $\mathrm{TG} \geq 8.33 \mathrm{mmol} / \mathrm{L}$ ) according to the guidelines by the National Cholesterol Education Program (NCEP) for metabolic syndrome. The relative odds ratios (OR) were expressed as exponentiation of the $\beta$ coefficient $(\operatorname{Exp}(\beta))$ with a $\mathrm{p}$-value. The OR was obtained for total samples, males and females. Statistical analyses were performed using SPSS software, where two-tailed and $p$-values $<0.05$ were considered statistically significant.

\section{Results}

Table 1 lists baseline characteristics of the population, compared on the basis of genetic variants (minor allele carrier, $n=71(62 \%)$ versus homozygous major allele, $n=45(38 \%)$ ). In general, the biochemical values of the two populations were comparable, although the minor allele carrier had significantly $(p=0.02)$ higher TG mean $(6.94 \mathrm{mmol} / \mathrm{L})$ as to homozygous major alleles $(5.27 \mathrm{mmol} / \mathrm{L})$. It should be noted that the male distribution was similar among two genetic groups (men: $40 \%$ versus $43 \%$ ) as using the chi-square test.

The study population was further divided on the basis of gender, and the comparison between genetic variants among each gender is detailed in Table 2. Interestingly, it can be seen that the TG difference is significantly higher in male $(p=0.02)$ with larger difference in mean, whereas the difference disappeared among female ( $p=$ 0.32 ).

The data, collected based on a random population who were presumed to be healthy and with no medical history, indicated that male naturally showed a significantly higher association between ADIPOR1 variants and TG compared to female. When life style variables were entered in the multivariate model with ADIPOR1, ADIPOR1 in male showed a significant and very high association with abnormal TG $(\operatorname{Exp}(\beta)=16.31, p=0.03)$.

\section{Discussion}

Based on the DPP population, Mather discovered that many of the adiponectin SNPs were associated with adiponectin concentration but none with diabetes incidence. ADIPOR1 SNP (rs1342387) had the most significant association with diabetes incidence, however, with ratio of only 1.18 [4]. Although the net effect being conveyed from the gene through adiponectin concentration onto diabetes risk was proportionally small in DPP, our findings highlighted the possibility of using same SNP to survey TG and taking gender into account could obtain much higher risk ratio $(\operatorname{Exp}(\beta) \sim 16)$. With appropriate target biomarkers, these genomic variants may be applied in early prevention of metabolic abnormality in specific gender during primary practice.

Mather et al. also found that non-genetic factors were more potent determinant in adiponectin concentration using multivariable analysis [3]. On the contrary, our finding in Table 3 demonstrated ADIPOR1 SNP can be a much stronger factor against hypertriglyceridemia than that compared to non-genetic factors in male. The difference between two findings might be the result of using different epidemiological method (randomized inferential survey versus placebo control), selecting down-stream biomarker (TG) instead of up-stream regulator (adiponectin), and taking gender into account. However, the future implications of our study have two limitations. First, this study was only conducted within Asian population. Second, we evaluated only the association with metabolic risk, not disease outcomes and thus it should be implied with other SNP studies for better screening model.

In conclusion, disease is often defined and risk calculated by various biochemical abnormality. Hypertriglyceridemia is a significant risk factor for both ischemic stroke [9] and coronary disease [10]. Our findings may suggest for practice of using ADIPOR1 SNP (rs1342387) to screen juvenile male with cerebrovascular disease 
Table 1. Baseline characteristics of the sample according to genetic variants.

\begin{tabular}{|c|c|c|c|c|}
\hline & \multicolumn{2}{|c|}{ ADIPOR1 } & & \\
\hline & $\mathrm{C} / \mathrm{C}$ & $\mathrm{T} / \mathrm{T}, \mathrm{T} / \mathrm{C}$ & & \\
\hline & $N=45$ & $\mathrm{~N}=71$ & & \\
\hline & Mean \pm SD & Mean \pm SD & $t$-value $/ \chi^{2}$ & $p$-value \\
\hline SBP (mmHg) & $124 \pm 16$ & $121 \pm 16$ & 0.71 & 0.48 \\
\hline DBP (mmHg) & $77 \pm 11$ & $75 \pm 11$ & 0.62 & 0.54 \\
\hline Chol (mmol/L) & $10.49 \pm 1.83$ & $10.77 \pm 1.78$ & -0.83 & 0.41 \\
\hline TG (mmol/L) & $5.27 \pm 2.66$ & $6.77 \pm 3.89$ & -2.47 & $0.02^{*}$ \\
\hline HDL (mmol/L) & $3.44 \pm 0.94$ & $3.33 \pm 0.89$ & 0.54 & 0.59 \\
\hline LDL (mmol/L) & $6.60 \pm 1.78$ & $6.83 \pm 1.78$ & -0.65 & 0.52 \\
\hline AC Sugar (mmol/L) & $5.27 \pm 1.28$ & $5.16 \pm 0.78$ & 0.80 & 0.43 \\
\hline HbA1C (\%) & $5.66 \pm 0.7$ & $5.6 \pm 0.57$ & 0.49 & 0.63 \\
\hline Age & $46 \pm 12$ & $36 \pm 73$ & 0.83 & 0.41 \\
\hline Male $^{\dagger}$ & $41 \%$ & $43 \%$ & 0.05 & 0.82 \\
\hline
\end{tabular}

${ }^{*}$ Statistical significance $p<0.05 ;{ }^{\dagger}$ Chi-square test.

Table 2. Comparison of the biomarkers in male and female according to ADIPOR1 genetic variants.

\begin{tabular}{|c|c|c|c|c|c|c|c|c|}
\hline & \multicolumn{4}{|c|}{ Male } & \multicolumn{4}{|c|}{ Female } \\
\hline & $\mathrm{C} / \mathrm{C}$ & $\mathrm{T} / \mathrm{T}, \mathrm{C} / \mathrm{T}$ & \multirow{2}{*}{$t$-value } & \multirow{2}{*}{$p$-value } & $\mathrm{C} / \mathrm{C}$ & $\mathrm{T} / \mathrm{T}, \mathrm{C} / \mathrm{T}$ & \multirow{2}{*}{$t$-value } & \multirow{2}{*}{$p$-value } \\
\hline & Mean \pm SD & Mean \pm SD & & & Mean \pm SD & Mean \pm SD & & \\
\hline Age & $47 \pm 13$ & $47 \pm 13$ & 0.17 & 0.87 & $46 \pm 10$ & $29 \pm 30$ & 0.87 & 0.39 \\
\hline SBP (mmHg) & $133 \pm 17$ & $126 \pm 17$ & 1.29 & 0.21 & $117 \pm 12$ & $117 \pm 15$ & 0.01 & 0.99 \\
\hline DBP (mmHg) & $82 \pm 9$ & $78 \pm 10$ & 1.60 & 0.12 & $72 \pm 10$ & $74 \pm 12$ & -0.52 & 0.60 \\
\hline Chol (mmol/L) & $10.55 \pm 1.78$ & $11.04 \pm 1.89$ & -0.95 & 0.35 & $10.43 \pm 2.00$ & $10.60 \pm 0.72$ & -0.28 & 0.78 \\
\hline TG (mmol/L) & $5.55 \pm 1.89$ & $7.94 \pm 4.77$ & -2.45 & $0.02^{*}$ & $5.16 \pm 3.16$ & $5.88 \pm 2.89$ & -1.00 & 0.32 \\
\hline $\mathrm{HDL}(\mathrm{mmol} / \mathrm{L})$ & $3.00 \pm 0.83$ & $2.83 \pm 0.67$ & 0.71 & 0.48 & $3.66 \pm 0.89$ & $3.66 \pm 0.89$ & -0.03 & 0.97 \\
\hline LDL (mmol/L) & $7.10 \pm 1.78$ & $7.49 \pm 1.78$ & -0.79 & 0.43 & $6.33 \pm 1.78$ & $6.33 \pm 1.55$ & -0.087 & 0.93 \\
\hline AC Sugar $(\mathrm{mmol} / \mathrm{L})$ & $5.11 \pm 0.56$ & $5.33 \pm 1$ & -0.73 & 0.47 & $5.44 \pm 1.61$ & $5.00 \pm 0.50$ & 1.24 & 0.23 \\
\hline HbA1C (\%) & $5.88 \pm 0.81$ & $5.68 \pm 0.80$ & 0.85 & 0.40 & $5.52 \pm 0.60$ & $5.54 \pm 0.30$ & -0.17 & 0.87 \\
\hline
\end{tabular}

*Statistical significance $p<0.05$.

Table 3. Multivariate logistic regression using TG $\geq 8.33 \mathrm{mmol} / \mathrm{L}$ as abnormal level.

\begin{tabular}{|c|c|c|c|c|c|c|c|c|c|}
\hline & \multicolumn{3}{|c|}{ Total } & \multicolumn{3}{|c|}{ Male } & \multicolumn{3}{|c|}{ Female } \\
\hline & $\operatorname{Exp}(ß)$ & Wald & $p$-value & $\operatorname{Exp}(ß)$ & Wald & $p$-value & $\operatorname{Exp}(ß)$ & Wald & $p$-value \\
\hline ADIPOR1 & 4.28 & 5.91 & $0.02^{*}$ & 16.31 & 4.86 & $0.03^{*}$ & 2.42 & 1.44 & 0.23 \\
\hline Stress score & 0.89 & 1.40 & 0.24 & 0.77 & 2.53 & 0.11 & 0.99 & 0.02 & 0.90 \\
\hline Diet behavior score & 1.00 & 0.01 & 0.93 & 1.03 & 0.19 & 0.66 & 0.96 & 0.45 & 0.50 \\
\hline Exercise score & 1.00 & 0.00 & 0.98 & 1.00 & 0.40 & 0.53 & 1.00 & 0.08 & 0.77 \\
\hline
\end{tabular}

* Statistical significance $p<0.05$. 
family history, and life style adjustment being employed upon minor allele carrier at young age. In the future, combining studies of high association between SNP and other abnormal biomarker may provide better implication for accessing disease risk among healthy individuals. Meantime, our study also provided clinical evidence for future bio-molecular studies regarding the gender effect of adiponectin receptor 1 in lipid metabolism.

\section{Acknowledgements}

This study was supported by Professor K. D. Yang’s research team and we also acknowledged the editorial assistance from Nigel Daly, Senior Editor of Asia Training Solutions Incorporation.

\section{References}

[1] Ginsberg, H.N., Zhang, Y.L. and Hernandez-Ono, A. (2005) Regulation of Plasma Triglycerides in Insulin Resistance and Diabetes [Review]. Archives of Medical Research, 36, 232-240. http://dx.doi.org/10.1016/j.arcmed.2005.01.005

[2] Shin, D.W., Lee, K.B., Seo, J.Y., Kim, J.S., Roh, H., Ahn, M.Y., et al. (2015) Association between Hypertriglyceridemia and Lacunar Infarction in Type 2 Diabetes Mellitus. Journal of Stroke and Cerebrovascular Diseases, 24, 18731878. http://dx.doi.org/10.1016/j.jstrokecerebrovasdis.2015.04.030

[3] Mather, K.J., Funahashi, T., Matsuzawa, Y., Edelstein, S., Bray, G.A., Kahn, S.E., et al. (2008) Adiponectin, Change in Adiponectin, and Progression to Diabetes in the Diabetes Prevention Program. Diabetes, 57, 980-986. http://dx.doi.org/10.2337/db07-1419

[4] Mather, K.J., Christophi, C.A., Jablonski, K.A., Knowler, W.C., Goldberg, R.B., Kahn S.E., et al. (2012) Common Variants in Genes Encoding Adiponectin (ADIPOQ) and Its Receptors (ADIPOR1/2), Adiponectin Concentrations, and Diabetes Incidence in the Diabetes Prevention Program. Diabetic Medicine, 29, 1579-1588. http://dx.doi.org/10.1111/j.1464-5491.2012.03662.x

[5] Olefsky, J., Farquhar, J.W. and Reaven, G.M. (1974) Sex Difference in the Kinetics of Triglyceride Metabolism in Normal and Hypertriglyceridaemic Human Subjects. European Journal of Clinical Investigation, 4, 121-127. http://dx.doi.org/10.1111/j.1365-2362.1974.tb02324.x

[6] Mauro, L., Pellegrino, M., De Amicis, F., Ricchio, E., Giordano, F., Rizza, P., et al. (2014) Evidences that Estrogen Receptor $\alpha$ Interferes with Adiponectin Effects on Breast Cancer Cell Growth. Cell Cycle, 13, 553-564. http://dx.doi.org/10.4161/cc.27455

[7] (2011) Taking Sex into Account in Medicine. The Lancet, 378, 1826.

[8] Crow, J.F. (1999) Hardy, Weinberg and Language impediments. Genetics, 152, 821-825.

[9] Laloux, P., Galanti, L. and Jamart, J. (2004) Lipids in Ischemic Stroke Subtypes. Acta Neurologica Belgica, 104, 1319.

[10] Sarwar, N., Danesh, J., Eiriksdottir, G., Sigurdsson, G., Wareham, N., Bingham, S., et al. (2007) Triglycerides and the Risk of Coronary Heart Disease: 10,158 Incident Cases among 262,525 Participants in 29 Western Prospective Studies. Circulation, 115, 450-458. http://dx.doi.org/10.1161/CIRCULATIONAHA.106.637793 